\title{
Computer Methods for Describing Groups from Binary Phenetic Data: Preliminary Summary and Editing of Data
}

\author{
CYNTHIA A. WALCZAK AND MICAH I. KRICHEVSKY \\ Microbial Systematics Section, National Institute of Dental Research, National Institutes of Health, \\ Bethesda, Maryland 20205
}

\begin{abstract}
Summarizing and editing binary phenetic data before further analysis are an accepted practice. The microbiologist tabulates basic information about a strain by feature matrix and uses this information as an aid in editing that matrix. Interactive computer technology can be used to aid in this process. Advantages of such computer-aided matrix editing include (i) preliminary analysis of the data, (ii) automation of routine operations, (iii) reduced costs of subsequent analyses, and (iv) improved results of subsequent analyses. The reduced costs and improved results are demonstrated by running a cluster analysis program on data before and after editing.
\end{abstract}

Computer manipulation of large masses of phenotypic data describing individual strains is a common part of many ecological and taxonomic studies. With bacteria, the data are most often recorded as three states of possibility: yes, no, or missing. The computer is used to perform analyses which range from simply making inverted lists (e.g., which strains have selected attributes in common) through feature frequencies of groups (e.g., from each marine sampling station, tabulate the numbers of strains able to degrade any of a number of hydrocarbons) to complete cluster analyses and inter- and intragroup statistics (which in turn can be used to calculate ecological diversity [2]).

The basic data structure used for these analyses may be considered (and in practice often is) a two-dimensional matrix or table of strains by features, with each possible cell occupied by a yes, a no, or a blank (3). Editing of the data matrix, before computer analysis, is done for economy and to remove extraneous data. The usual process involves either (i) manual editing and re-entry of data or (ii) using the computer system's editing capability. The first method is cumbersome and error prone; it should be avoided when possible. The second method is preferred because it works with the data file already in the computer. However, general-purpose text editors have limited decision-making capabilities. Thus, most of the decisions to be made on a microbial data matrix, no matter how mechanical the logic, still must be done on a cumbersome, custom basis by the microbiologist. After analysis of the most common editing operations performed by microbiologists, we evolved interactive computer techniques which tabulate basic information about the strains and features, edit the data according to user-set criteria, reformat the matrix, and inform the user of all decisions made during editing. Although the specific program runs under the MVS-TSO system of the IBM 370 complex at the National Institutes of Health, it should work on any IBM 370 computer with a similar operating system. Further, the logic of the operations is adaptable to most time-sharing computer environments.

\section{MATERIALS AND METHODS}

Program functions. The following functions are those selected as useful in editing of phenetic data. The first four are considered mandatory, whereas the last seven are often desirable.

(i) Tabulate the number of positive, negative, and missing items by feature or strain or both.

(ii) Calculate and print tables of frequency of positive, negative, and missing items by feature or strain or both. (Note: positive frequency is calculated as: 100 $\times$ positive items $/($ positive + negative items); negative frequency $=100 \times$ negative items $/$ (positive + negative items); frequency of missing items $=100 \times$ missing items $/$ (positive + negative + missing items).

(iii) Delete features or strains (or both) based on user-set separate parameters to delete any feature or strain desired.

(iv) Delete strains or features (or both) by user-set minimum thresholds for the percent positive frequency above which items will be deleted, the minimum threshold for negative frequency, and for missing data.

(v) List a summary of deletions.

(vi) Change the symbols that represent positive, negative, or missing items in the data matrix.

(vii) Compress empty space after deletion, and print a table of new column positions versus old.

(viii) Give rank of strains or features, or both, by positive frequency.

(ix) Rearrange data in order of positive-strain frequency. 
Astrain \% SIMIIARITY

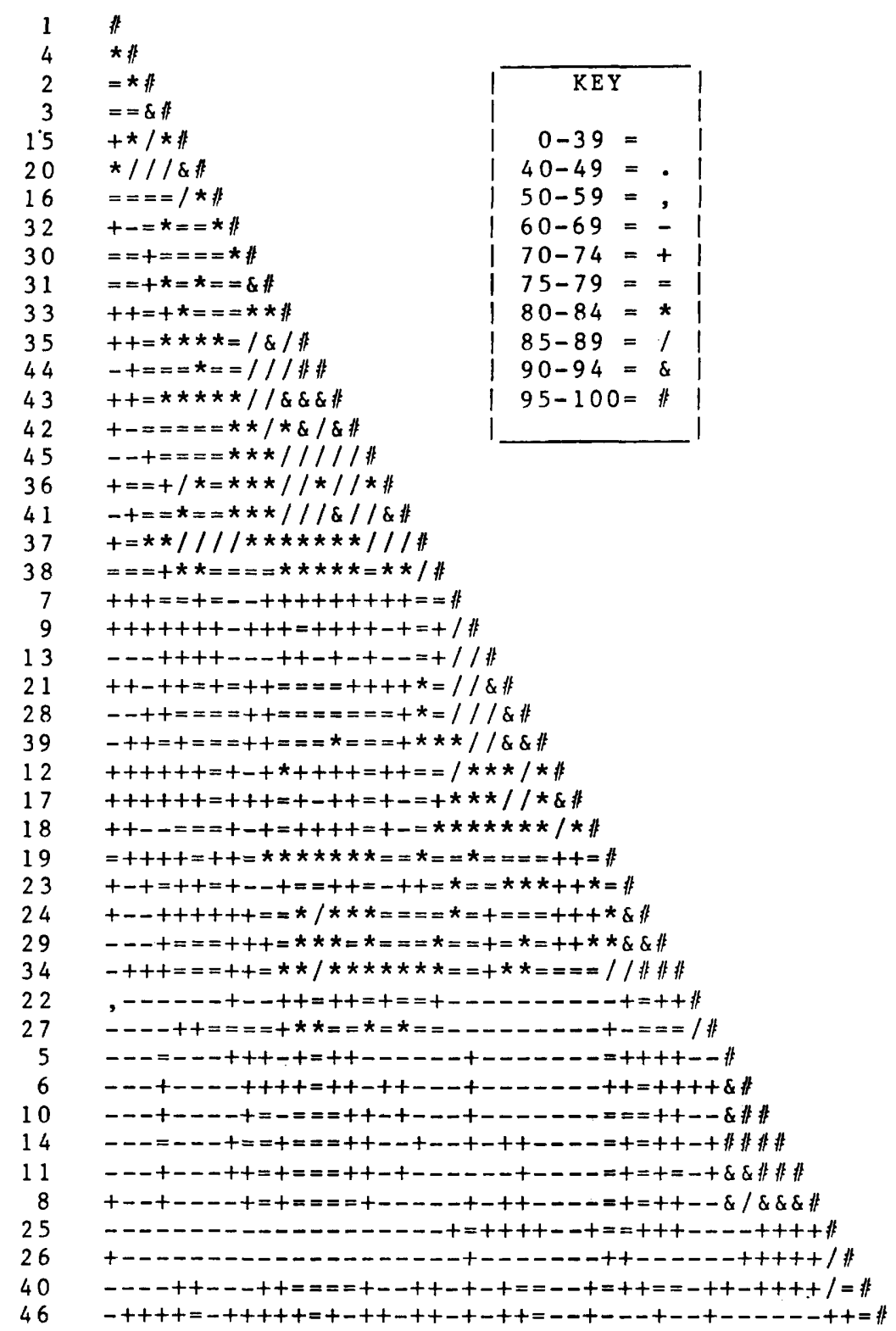

Frg. 1. (A) Complete similarity triangle resulting from cluster analysis of an unedited data matrix. (B) Triangle of all interstrain similarities greater than $80 \%$ resulting from cluster analysis of an unedited data matrix. The analysis is the same as in (A).

(x) Accept data in either collated or decollated format, and change data to either format for input into programs with specific requirements.

(xi) Make multiple passes.

Cluster analysis. To demonstrate the effect of editing a matrix on further analysis, cluster analyses were performed. The programs used are modified versions of TAXAN5, developed by P. H. A. Sneath and M. J. Sackin under the auspices of the Medical Research Council of Great Britain. The similarity coefficients used were simple matching and Jaccard. The clustering method was unweighted average linkage (4). 
BSTRAIN \% SIMILARITY

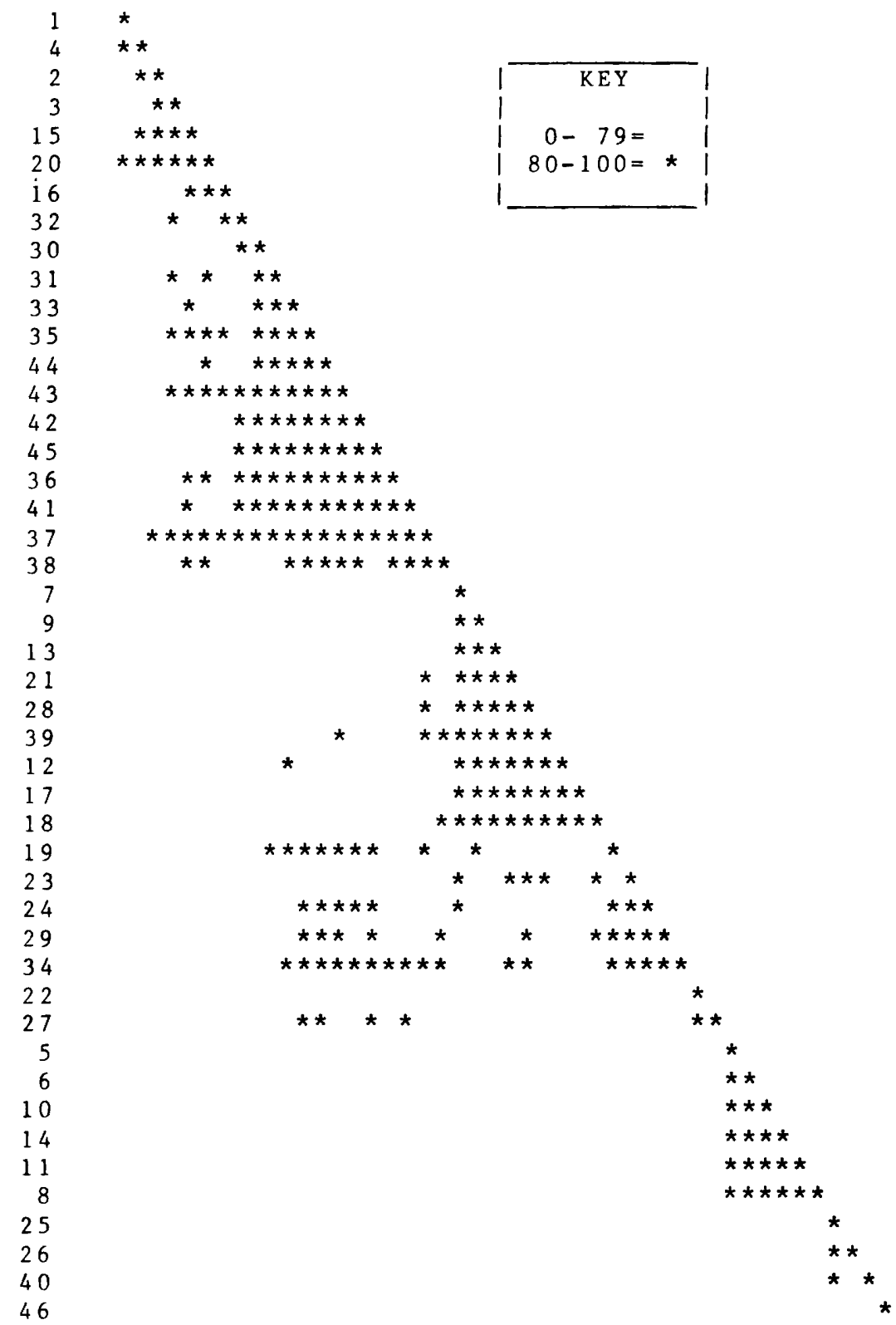

Fig. 1B

\section{RESULTS AND DISCUSSION}

An interactive, easily used editing program gives the microbiologist the capability of obtaining immediate summary information about a strain by feature matrix, which can be used as an aid in editing the matrix for further analysis.
The advantages of such interactive matrix editing are (i) the preliminary analysis of the data, (ii) automation of routine operations (hence, reduced labor), (iii) reduced costs of subsequent analyses, and (iv) improvement of subsequent analyses.

The preliminary data analysis, in the form of 


\section{ASTRAIN \% SIMILARITY}

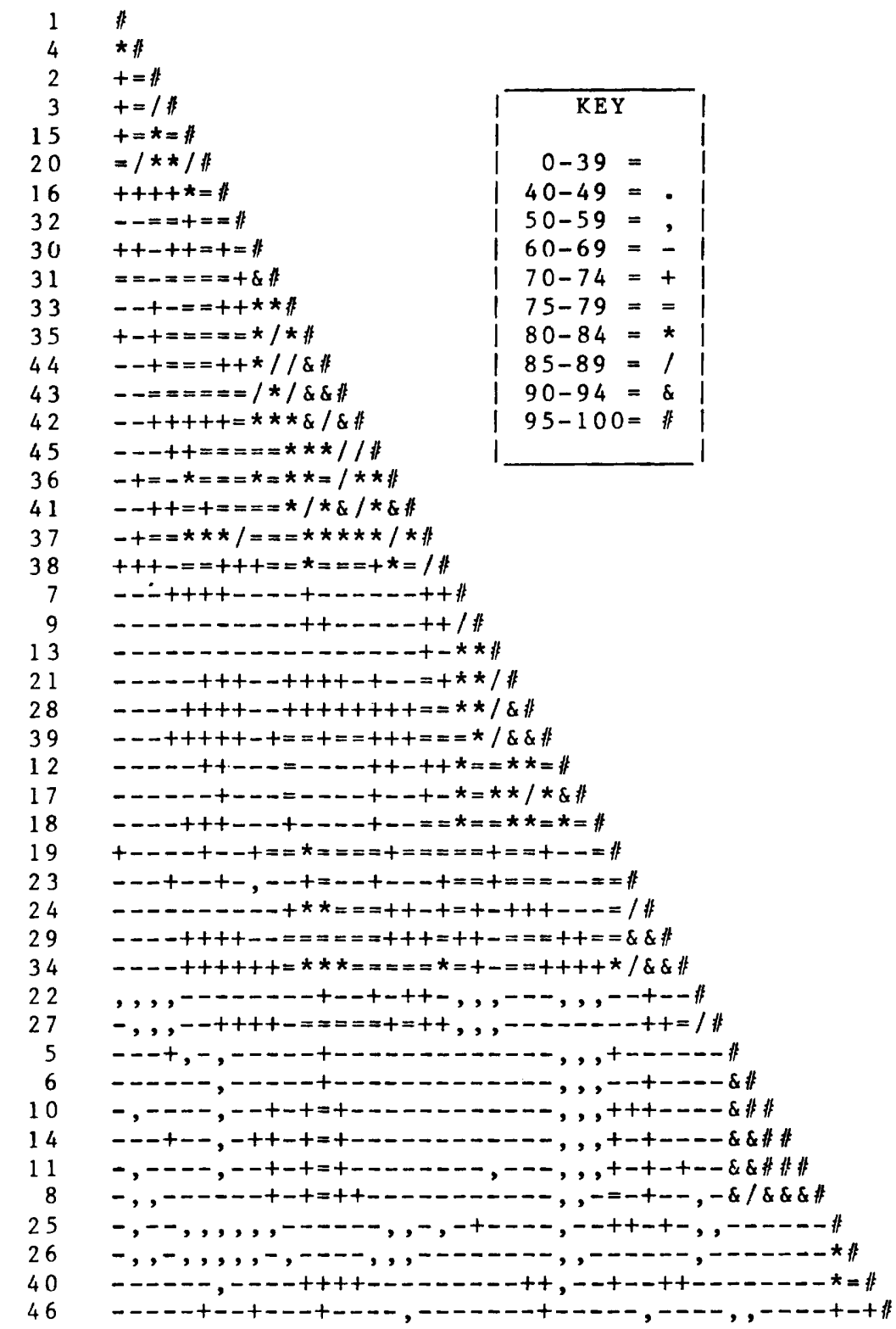

FIG. 2. (A) Complete similarity triangle resulting from editing the data before cluster analysis. (B) Triangle of all interstrain similarities greater than $80 \%$ resulting from editing the data before cluster analysis. The analysis is the same as in $(A)$.

summary tables, aids the microbiologist in editing the strain by feature matrix. The summary tables developed can be listed at the terminal. Based on the initial summary, thresholds can be set to eliminate strains or features or both from the matrix. In practice, the thresholds which are varied the most are those for missing scores. Many matrices have incomplete data which are not distributed randomly. The threshold level is varied according to the extent of the omitted data.

The user can obtain a preliminary analysis of 
BSTRAIN \% SIMILARITY

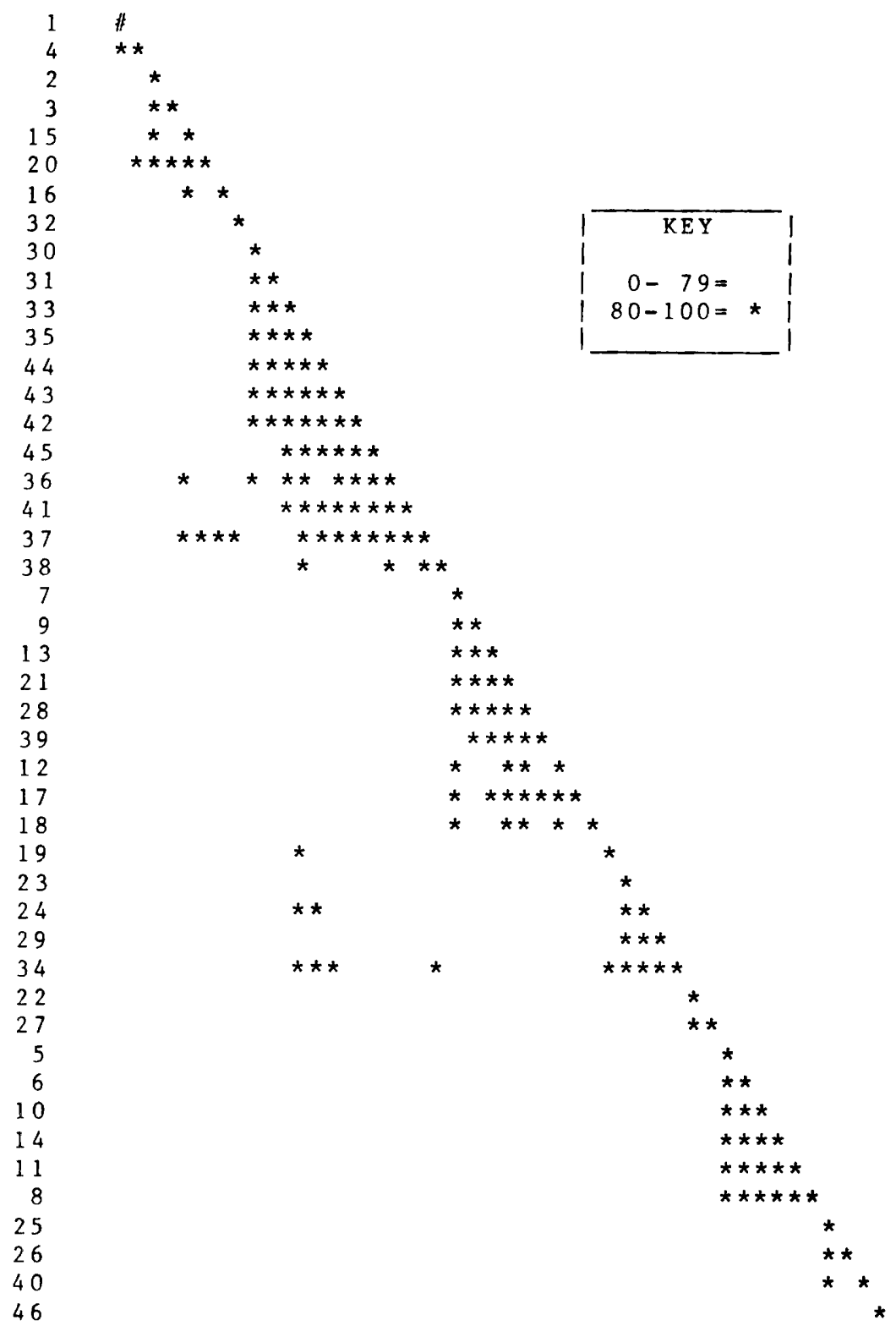

FIG. 2B

the matrix. Using this analysis, the user can delete strains or features, or both, and then obtain another analysis, perform further deletions, etc. Reentering the program with the original matrix can be done to experiment with different threshold levels. The various analytic capabilities of the program have their own merits. However, the primary purpose for their in- clusion is to aid in judging the threshold levels to be set for strain or feature elimination.

The most obvious advantage of an editing program is the automation of the routine operations normally performed in conditioning data before analysis, thereby markedly reducing the required labor costs. Other costs reduced by these procedures are for computer time and 
space needed for computation of results. As larger matrices are analyzed, the economic costs can be burdensome and the space requirements can be so large as to prevent the computation without drastic reprogramming. Whereas, in theory, any size of matrix can be analyzed, the prohibitive cost and time lag for reprogramming make it impractical to implement the theory.

The benefits of row and column elimination in terms of computer time (costs) are shown in Table 1 for a series of cluster analyses. For example, increasing the number of strains from 293 to 445 (runs 1 and 2) caused a disproportionately large increase in cost and, even more, in computer time used. More than doubling the number of features from 85 to 216 (runs 3 and 4) did not result in an equivalent rise in costs or computer time used (although the rise is still significant). The cost of editing is insignificant compared with the savings realized in the course of further analyses. (The costs shown are meant only to illustrate the principles involved.)

Perhaps the most important benefit of editing is the improvement in analytic results obtained by removing meaningless strains and features. These are ones that are redundant or have insufficient data, or are inappropriate for some other reason $(1,4)$. Again, using the example of cluster analysis, more discrimination of groups must occur when features are removed from the matrix that lack discriminating power; i.e., those that are essentially either all positive or all negative for every strain in the study.

To illustrate the improved analytic results obtained with editing, we analyzed the clustering behavior of 46 strains of anaerobic and facultatively anaerobic coryneforms. (The data were kindly supplied by N. S. Hunter and J. K. Read.) A cluster analysis was performed on the original 46 strains by a 138 -feature matrix (Fig. $1 \mathrm{~A}$ and B). Then the matrix was edited as follows. The thresholds set for automatic elimination were $99 \%$ positive, $99 \%$ negative, and $90 \%$ missing data for features. Twenty-three features were elimi-

TABLE 1. Computer resources used during cluster analysis of various sizes of data matrices

\begin{tabular}{ccccr}
\hline $\begin{array}{c}\text { Run } \\
\text { no. }\end{array}$ & $\begin{array}{c}\text { No. of } \\
\text { strains }\end{array}$ & $\begin{array}{c}\text { No. of } \\
\text { features }\end{array}$ & $\begin{array}{c}\text { Computer } \\
\text { time } \\
\text { (s) }\end{array}$ & Cost \\
\hline 1 & 445 & 129 & 1,918 & $\$ 165.24$ \\
2 & 293 & 129 & 555 & 59.19 \\
& & & & \\
3 & 280 & 216 & 700 & 60.28 \\
4 & 277 & 85 & 362 & 37.54 \\
& & & & \\
5 & 301 & 237 & 855 & 77.66 \\
6 & 247 & 244 & 571 & 49.61 \\
\hline
\end{tabular}

nated. The thresholds for strains were set such that none were eliminated ( $101 \%$ positive, $101 \%$ negative, and $101 \%$ missing data).

After cluster analysis of the edited data by the simple matching coefficient and average linkage sorting, the result (Fig. 2A and B) was compared with that obtained without prior editing. The first conclusion is that the order of the strains was identical between the two analyses. Comparison of the similarity triangles from each of the analyses (Fig. 1A and 2A) indicates that the clusters are more distinct after editing. That is, the boundaries of the internal "triangles" lying along the hypotenuse of the total triangle more closely approximate the desired theoretical straight lines at all levels of similarity. This is most clearly illustrated by changing the symbols of the triangles so that blank spaces denote similarities below $80 \%$ and an asterisk denotes those of $80 \%$ or more (Fig. $1 \mathrm{~B}$ and $2 \mathrm{~B}$ ). The unedited data matrix results in a $17 \%$ minimum similarity among all strains (by simple matching coefficient) because 23 features are either all positive or all negative in the matrix $[(23 / 138)$ $\times 100=17 \%$ ]. Elimination of these 23 features avoids scale compression and increases resolution. Similar results are obtained on the same data when the Jaccard similarity coefficient is used in the analyses. Thus, the desired result of improved analyses is realized.

With either the simple matching coefficient or the Jaccard coefficient of similarity, the position of a strain (and hence its place in the resulting taxonomy) will not change if the thresholds for positive and negative feature frequencies are set at $100 \%$. Only the numerical value of similarity is lowered. (With the Jaccard coefficient, negative matches are ignored, so a threshold of $100 \%$ negative has no effect on the similarity value.) Thresholds below $100 \%$ will "improve" clustering at the expense of the features unique to small groups. That is, a group of strains having features different from all other groups may go undetected as the thresholds are lowered below $100 \%$ positive or negative. As thresholds for positive- and negative-feature frequencies approach $50 \%$, groups will usually become larger and more clearly defined. This effect may be used in finding efficient features for incorporation into identification schema.

Since it is seldom of importance to eliminate strains on the basis of frequency of positive or negative reactions, these threshold settings would usually be set at $101 \%$. (no elimination).

The threshold for missing data is of importance in eliminating strains which act as distorting bridges between clusters. Such strains tend to "float" as differing similarity coefficients are 
used. In practice, we find that a threshold for missing strain data between 10 and $25 \%$ is satisfactory for most sets of data. A threshold of 90\% missing data for features is used. A large amount of missing data for a given feature is not serious provided not too many features have such a dearth of data.

Although we used the example of cluster analysis, many other analytic procedures will show many of the same effects of editing. A correlation matrix or variance calculation would be similarly affected by scale compression if the data are not edited. Since editing of matrices is both common and labor intensive, automation by interactive computer methodology has proved most useful.

Listings and documentation of the EDITMAT program are available from us.

\section{REPRINT REQUESTS}

Address reprint requests to: Dr. Micah I. Krichevsky, Microbial Systematics Section, National Institute of Dental Research, National Institutes of Health, Bethesda, MD 20205.

\section{LITERATURE CITED}

1. Colwell, R. R. 1970. Collecting the data, p. 4-21. In W. R. Lockhart and J. Liston (ed.), Methods for numerical taxonomy. American Society for Microbiology, Bethesda, Md.

2. Kaneko, T., R. M. Atlas, and M. I. Krichevsky. 1977. Diversity of bacterial populations in the Beaufort Sea. Nature (London) 270:596-599.

3. Krichevsky, M. I. 1977. Coding and manipulation of strain data, p. 150-156. In Proceedings of the III International Conference on Culture Collections. University of Bombay, Bombay.

4. Sneath, P. H. A., and R. R. Sokal. 1973. Numerical taxonomy. W.H. Freeman and Co., San Francisco. 